\title{
Discovery of potent antivirals against amantadine-resistant influenza A viruses by targeting the M2-S31N proton channel
}

\author{
Fang Li, ${ }^{\dagger \dagger}$ Chunlong Ma, ${ }^{\dagger, \dagger}$ Yanmei Hu, ${ }^{\dagger, \dagger}$ Yuanxiang Wang, ${ }^{\dagger}$ Jun Wang ${ }^{*, \dagger,}$
}

†Department of Pharmacology and Toxicology, College of Pharmacy, The University of Arizona, Tucson, Arizona 85721, United States

${ }^{\sharp}$ BIO5 Institute, The University of Arizona, Tucson, Arizona, 85721, United States

*Corresponding author:

Jun Wang, Tel: 520-626-1366, Fax: 520-626-0749, email: junwang@pharmacy.arizona.edu

\section{$\underline{\text { Chemistry }}$}

All chemicals were purchased from commercial vendors and used without further purification unless otherwise noted. ${ }^{1} \mathrm{H}$ and ${ }^{13} \mathrm{C}$ NMR spectra were recorded on a Bruker400 NMR spectrometer. Chemical shifts are reported in parts per million referenced with respect to residual solvent $\left(\mathrm{CD}_{3} \mathrm{OD}\right) 3.31 \mathrm{ppm}$ and (Chloroform-d) $7.24 \mathrm{ppm}$ or from internal standard tetramethylsilane (TMS) $0.00 \mathrm{ppm}$. The following abbreviations were used in reporting spectra: s, singlet; d, doublet; t, triplet; q, quartet; m, multiplet; dd, doublet of doublets; ddd, doublet of doublet of doublets. High resolution mass spectra were obtained using positive ESI method for all the compounds and spectra were obtained with an Ion Cyclotron Resonance (ICR) spectrometer. All reactions were carried out under $\mathrm{N}_{2}$ atmosphere, unless otherwise stated. HPLC-grade solvents were used for all reactions. Flash column chromatography was performed using silica gel (230-400 mesh, Merck). Low-resolution mass spectra were obtained using an ESI technique on a 3200 Q Trap LC/MS/MS system (Applied Biosystems). The purity was assessed by using 
Shimadzu LC-MS with Waters XTerra MS C-18 column (part \#186000538), $50 \times 2.1$ $\mathrm{mm}$, at a flow rate of $0.3 \mathrm{~mL} / \mathrm{min} ; \lambda=250$ and $220 \mathrm{~nm}$; mobile phase $\mathrm{A}, 0.1 \%$ formic acid in $\mathrm{H}_{2} \mathrm{O}$, and mobile phase $\mathrm{B}^{\prime}, 0.1 \%$ formic in $60 \%$ isopropanol, $30 \% \mathrm{CH}_{3} \mathrm{CN}$ and $9.9 \%$ $\mathrm{H}_{2} \mathrm{O}$. All compounds submitted for testing in TEVC assay and plaque reduction assay were confirmed to be $>95.0 \%$ purity by LC-MS traces. All compounds were characterized by proton NMR and MS, and selected compounds were also characterized by carbon NMR.

\section{$\underline{\text { Synthesis Procedures }}$}

Compounds 9, 12, 15, and 19 were synthesized according to method I.

\section{Method I: General procedure of reductive amination (Scheme 1):}

Adamantane or 3-amino-1-adamantanol (1 equiv.) and aldehyde (1 equiv.) were mixed with $2 \mathrm{~mL}$ of titanium (IV) isopropoxide. The resulting slurry was heated in microwave at $120{ }^{\circ} \mathrm{C}$ for 30 minutes. Then the solution was cooled down and $\mathrm{CH}_{3} \mathrm{OH}(10 \mathrm{~mL})$ was added. The mixture was cooled down to $0{ }^{\circ} \mathrm{C}$ using ice bath, then $\mathrm{NaBH}_{4}$ (4 equiv.) was added portionwise in 10 minutes. The solution was subsequently warmed to room temperature and stirred for 4 hours. The reaction was quenched with $1 \mathrm{M} \mathrm{NaOH}$ and filtered through celite. The filtrate was concentrated under reduced pressure and was purified by silica gel flash column chromatography $\left(5-10 \% \mathrm{CH}_{3} \mathrm{OH} / \mathrm{CH}_{2} \mathrm{Cl}_{2}\right)$ to give the final product.

Compounds 10a-i, 13a-f, 16a-f, and 20a-f were synthesized according to method II. Method II: General procedure of Buchwald-Hartwig coupling (Scheme 1):

To a solution of adamantane substituted bromide and chloride (1 equiv.) in toluene ( $2 \mathrm{~mL})$ was added amine (1.2 equiv.), sodium tert-butoxide (1.4 equiv.), and 2,2'- 
bis(diphenylphosphino)-1,1'-binaphthyl (15\% mol). The mixture was purged with dry nitrogen for 5 minutes. Then tris(dibenzylideneacetone)dipalladium (5\% mol) was added and the solution was heated in microwave at $100{ }^{\circ} \mathrm{C}$ for 30 minutes. The solvent was removed under reduced pressure, and the resulting residue was extracted with dichloromethane and water. The organic layer was separated, dried over anhydrous magnesium sulfate, filtered and concentrated under reduced pressure. The mixture was then purified by silica gel flash column chromatography $\left(0-10 \% \mathrm{CH}_{3} \mathrm{OH} / \mathrm{CH}_{2} \mathrm{Cl}_{2}\right)$ to give the final product.

\section{Characterizations}

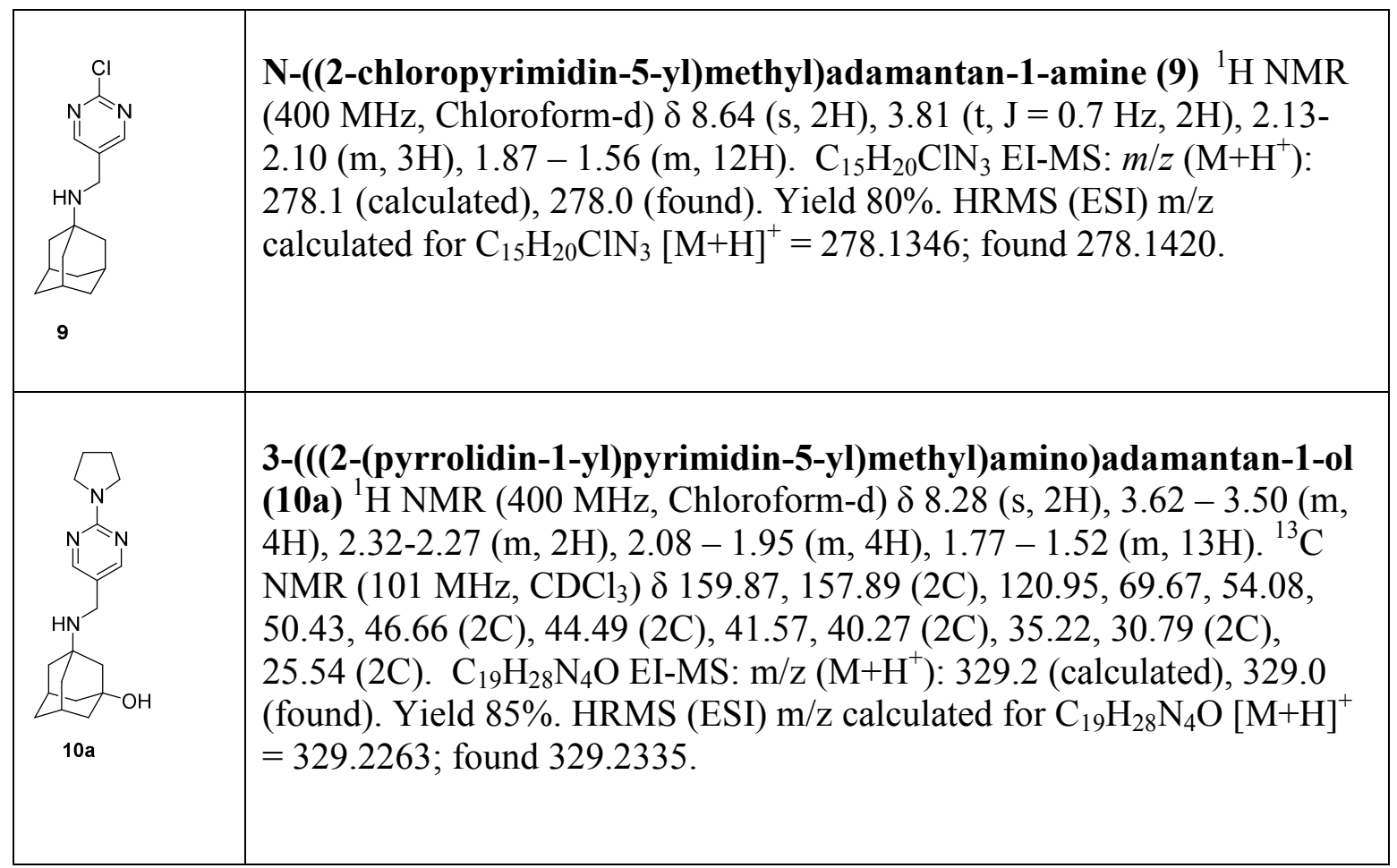




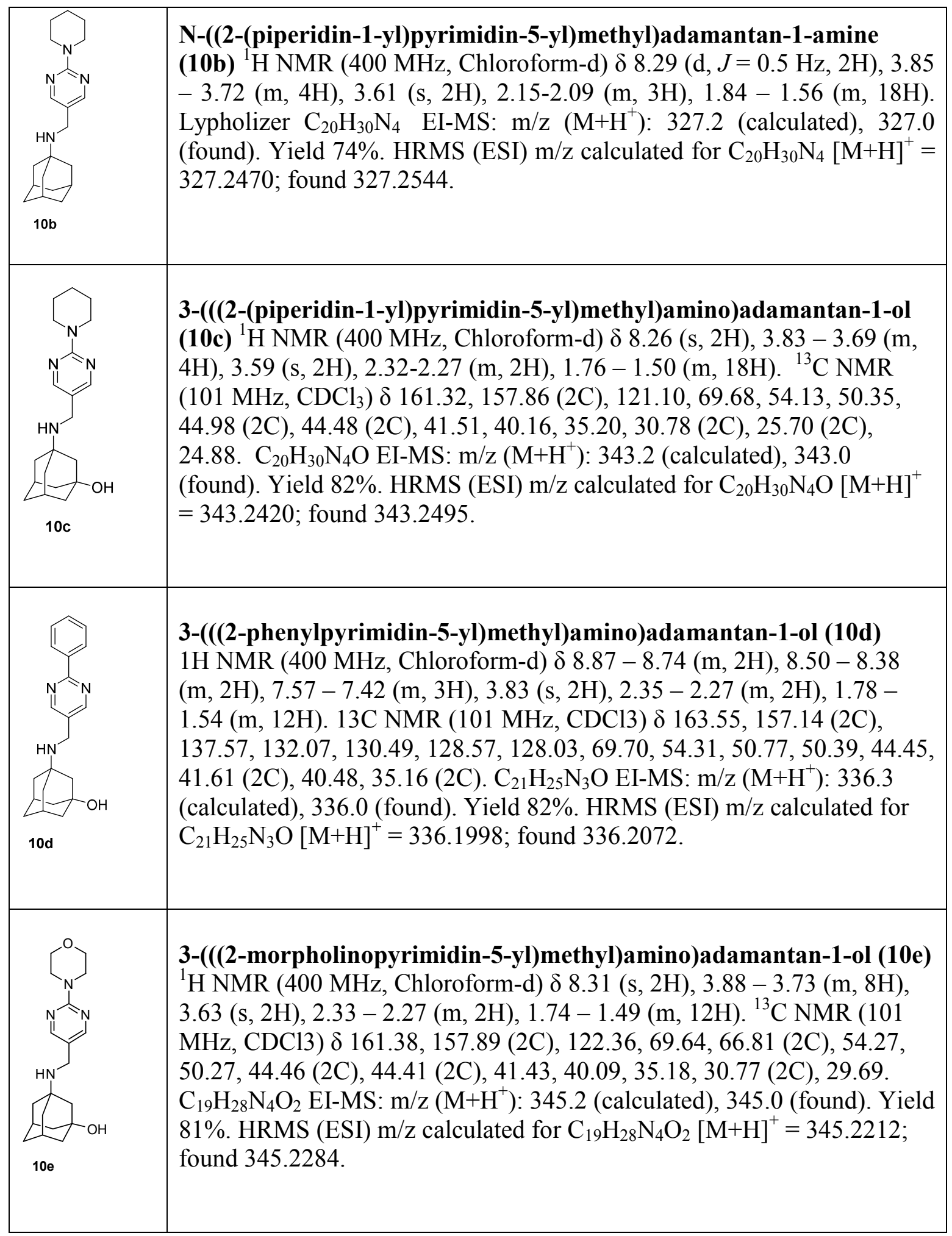




\begin{tabular}{|c|c|}
\hline & $\begin{array}{l}\text { N-((2-(azepan-1-yl)pyrimidin-5-yl)methyl)adamantan-1-amine (10f) } \\
{ }^{1} \mathrm{H} \text { NMR }(400 \mathrm{MHz}, \mathrm{Chloroform}-\mathrm{d}) \delta 8.47(\mathrm{~s}, 2 \mathrm{H}), 3.73(\mathrm{~s}, 2 \mathrm{H}), 3.72- \\
3.67(\mathrm{~m}, 4 \mathrm{H}), 2.16-2.11(\mathrm{~m}, 3 \mathrm{H}), 1.96-1.91(\mathrm{~m}, 5 \mathrm{H}), 1.78-1.72(\mathrm{~m}, 5 \mathrm{H}) \text {, } \\
1.70-1.67(\mathrm{~m}, 6 \mathrm{H}), 1.56-1.51(\mathrm{~m}, 5 \mathrm{H}) .{ }^{13} \mathrm{C} \mathrm{NMR}(125 \mathrm{MHz}, \mathrm{Chloroform}- \\
\text { d) } \delta 160.13,159.68,111.85,57.86,47.41,38.53,38.33,35.71,29.12, \\
27.77,27.38 . \mathrm{C}_{21} \mathrm{H}_{32} \mathrm{~N}_{4} \mathrm{EI}-\mathrm{MS}: \mathrm{m} / \mathrm{z}\left(\mathrm{M}+\mathrm{H}^{+}\right): 341.3(\text { calculated }), 341.0 \\
\text { (found). Yield } 72 \% \text {. HRMS }(\mathrm{ESI}) \mathrm{m} / \mathrm{z} \text { calculated for } \mathrm{C}_{21} \mathrm{H}_{32} \mathrm{~N}_{4}[\mathrm{M}+\mathrm{H}]^{+}= \\
341.2627 \text {; found } 341.2701 .\end{array}$ \\
\hline 2 & $\begin{array}{l}\text { 3-(((2-(azepan-1-yl)pyrimidin-5-yl)methyl)amino)adamantan-1-ol } \\
(\mathbf{1 0 g}){ }^{1} \mathrm{H} \text { NMR }(400 \mathrm{MHz}, \mathrm{Chloroform}-\mathrm{d}) \delta 8.59(\mathrm{~d}, J=0.7 \mathrm{~Hz}, 2 \mathrm{H}), 3.77 \\
(\mathrm{~s}, 2 \mathrm{H}), 2.87-2.74(\mathrm{~m}, 4 \mathrm{H}), 2.27(\mathrm{~s}, 2 \mathrm{H}), 1.71-1.50(\mathrm{~m}, 20 \mathrm{H}) . \text { Low } \\
\text { conc. Redo } \mathrm{C}_{21} \mathrm{H}_{32} \mathrm{~N}_{4} \mathrm{O} \mathrm{EI}-\mathrm{MS}: \mathrm{m} / \mathrm{z}\left(\mathrm{M}+\mathrm{H}^{+}\right): 357.3\left(\text { calculated }^{+}, 357.0\right. \\
\text { (found). Yield } 73 \% \text {. HRMS }(\mathrm{ESI}) \mathrm{m} / \mathrm{z} \text { calculated for } \mathrm{C}_{21} \mathrm{H}_{32} \mathrm{~N}_{4} \mathrm{O}[\mathrm{M}+\mathrm{H}]^{+} \\
=357.2576 \text {; found } 357.2651 .\end{array}$ \\
\hline$-\underbrace{}_{10 \mathrm{~h}}$ & $\begin{array}{l}\text { N-((2-(azocan-1-yl)pyrimidin-5-yl)methyl)adamantan-1-amine (10h) } \\
{ }^{1} \mathrm{H} \text { NMR }(400 \mathrm{MHz} \text {, Chloroform-d) } \delta 8.48(\mathrm{dd}, J=1.7,0.9 \mathrm{~Hz}, 1 \mathrm{H}), 3.99 \\
(\mathrm{s}, 2 \mathrm{H}), 3.70(\mathrm{~s}, 1 \mathrm{H}), 2.83(\mathrm{~d}, J=5.0 \mathrm{~Hz}, 4 \mathrm{H}), 2.19-2.07(\mathrm{~m}, 3 \mathrm{H}), 1.77- \\
1.55(\mathrm{~m}, 22 \mathrm{H}) . \text { Resynthesize } \mathrm{C}_{22} \mathrm{H}_{34} \mathrm{~N}_{4} \mathrm{EI}-\mathrm{MS}: \mathrm{m} / \mathrm{z}\left(\mathrm{M}+\mathrm{H}^{+}\right): 355.5 \\
\text { (calculated), } 355.0 \text { (found). Yield } 80 \% \text {. HRMS }(\mathrm{ESI}) \mathrm{m} / \mathrm{z} \text { calculated for } \\
\mathrm{C}_{22} \mathrm{H}_{34} \mathrm{~N}_{4}[\mathrm{M}+\mathrm{H}]^{+}=355.2783 \text {; found } 355.2855 \text {. }\end{array}$ \\
\hline$[D$ & $\begin{array}{l}\text { 3-(((2-(azocan-1-yl)pyrimidin-5-yl)methyl)amino)adamantan-1-ol } \\
\text { (10i) }{ }^{1} \mathrm{H} \text { NMR }(400 \mathrm{MHz} \text {, Chloroform-d) } \delta 8.62(\mathrm{~s}, 2 \mathrm{H}), 3.79(\mathrm{~s}, 2 \mathrm{H}) \text {, } \\
2.87-2.78(\mathrm{~m}, 4 \mathrm{H}), 2.30(\mathrm{~s}, 2 \mathrm{H}), 1.75-1.43(\mathrm{~m}, 22 \mathrm{H}) \text {. Resynthesize } \\
\mathrm{C}_{22} \mathrm{H}_{34} \mathrm{~N}_{4} \mathrm{O} \text { EI-MS: } \mathrm{m} / \mathrm{z}\left(\mathrm{M}+\mathrm{H}^{+}\right): 371.3(\text { calculated }), 371.0 \text { (found). Yield } \\
72 \% \text {. HRMS }(\mathrm{ESI}) \mathrm{m} / \mathrm{z} \text { calculated for } \mathrm{C}_{22} \mathrm{H}_{34} \mathrm{~N}_{4} \mathrm{O}[\mathrm{M}+\mathrm{H}]^{+}=371.2733 ; \\
\text { found } 371.2805 \text {. }\end{array}$ \\
\hline
\end{tabular}




\begin{tabular}{|c|c|}
\hline$E$ & $\begin{array}{l}\text { N-((6-bromopyridin-3-yl)methyl)adamantan-1-amine (12) } \\
{ }^{1} \mathrm{H} \mathrm{NMR}(499 \mathrm{MHz}, \mathrm{Chloroform}-\mathrm{d}) \delta 8.29(\mathrm{~d}, J=2.5 \mathrm{~Hz}, 1 \mathrm{H}), 7.59(\mathrm{dd}, J \\
=8.2,2.5 \mathrm{~Hz}, 1 \mathrm{H}), 7.39(\mathrm{~d}, J=8.1 \mathrm{~Hz}, 1 \mathrm{H}), 3.72(\mathrm{~s}, 2 \mathrm{H}), 2.10-2.04(\mathrm{~m}, \\
3 \mathrm{H}), 1.73-1.55(\mathrm{~m}, 12 \mathrm{H}) .{ }^{13} \mathrm{C} \mathrm{NMR}(125 \mathrm{MHz}, \mathrm{Chloroform}-\mathrm{d}) \delta 150.02, \\
140.36,138.97,136.68,127.84,51.35,42.92,41.86,36.75,29.68 . \\
\mathrm{C}_{16} \mathrm{H}_{21} \mathrm{BrN}_{2} \text { EI-MS: } \mathrm{m} / \mathrm{z}\left(\mathrm{M}+\mathrm{H}^{+}\right): 321.1(\text { calculated }), 321.0 \text { (found). } \\
\text { Yield } 83 \% \text {. HRMS }(\mathrm{ESI}) \mathrm{m} / \mathrm{z} \text { calculated for } \mathrm{C}_{16} \mathrm{H}_{21} \mathrm{BrN}_{2}[\mathrm{M}+\mathrm{H}]^{+}= \\
\text {321.0888; found } 321.1588 .\end{array}$ \\
\hline$\underbrace{H N^{\prime}}_{13 a}$ & $\begin{array}{l}\text { N-((6-(piperidin-1-yl)pyridin-3-yl)methyl)adamantan-1-amine (13a) } \\
{ }^{1} \mathrm{H} \mathrm{NMR}(400 \mathrm{MHz}, \text { Chloroform-d) } \delta 8.13(\mathrm{dd}, J=2.5,0.7 \mathrm{~Hz}, 1 \mathrm{H}), 7.59 \\
(\mathrm{dd}, J=8.8,2.5 \mathrm{~Hz}, 1 \mathrm{H}), 6.63(\mathrm{dd}, J=8.8,0.8 \mathrm{~Hz}, 1 \mathrm{H}), 3.68(\mathrm{~s}, 2 \mathrm{H}) \\
3.52-3.47(\mathrm{~m}, 4 \mathrm{H}), 2.11(\mathrm{~s}, 3 \mathrm{H}), 1.90-1.55(\mathrm{~m}, 18 \mathrm{H}) .{ }^{13} \mathrm{C} \mathrm{NMR}(125 \\
\mathrm{MHz}, \mathrm{Chloroform}-\mathrm{d}) \delta 159.31,148.18,138.60,107.07,46.56,41.99, \\
36.63,29.66,25.63,24.85 . \mathrm{C}_{21} \mathrm{H}_{31} \mathrm{~N}_{3} \mathrm{EI}-\mathrm{MS}: \mathrm{m} / \mathrm{z}\left(\mathrm{M}+\mathrm{H}^{+}\right): 326.2 \\
(\text { calculated }), 326.0 \text { (found). Yield } 82 \% . \mathrm{HRMS}(\mathrm{ESI}) \mathrm{m} / \mathrm{z} \text { calculated for } \\
\mathrm{C}_{21} \mathrm{H}_{31} \mathrm{~N}_{3}[\mathrm{M}+\mathrm{H}]^{+}=326.2518 \text {; found } 326.2590 .\end{array}$ \\
\hline 4 & $\begin{array}{l}\text { 3-(((6-(piperidin-1-yl)pyridin-3-yl)methyl)amino)adamantan-1-ol } \\
(\mathbf{1 3 b}){ }^{1} \mathrm{H} \text { NMR }(400 \mathrm{MHz}, \mathrm{Chloroform}-\mathrm{d}) \delta 8.31(\mathrm{~d}, J=2.4 \mathrm{~Hz}, 1 \mathrm{H}), 7.60 \\
(\mathrm{dd}, J=8.2,2.4 \mathrm{~Hz}, 1 \mathrm{H}), 7.42(\mathrm{~d}, J=8.2 \mathrm{~Hz}, 1 \mathrm{H}), 3.74(\mathrm{~s}, 2 \mathrm{H}), 2.85-2.73 \\
(\mathrm{~m}, 4 \mathrm{H}), 2.27(\mathrm{~s}, 2 \mathrm{H}), 1.72-1.44(\mathrm{~m}, 18 \mathrm{H}) . \mathrm{C}_{21} \mathrm{H}_{31} \mathrm{~N}_{3} \mathrm{O} \text { EI-MS: } \mathrm{m} / \mathrm{z} \\
\left(\mathrm{M}+\mathrm{H}^{+}\right): 342.2(\text { calculated }), 342.0 \text { (found). Yield } 76 \% \text {. HRMS }(\mathrm{ESI}) \mathrm{m} / \mathrm{z} \\
\text { calculated for } \mathrm{C}_{21} \mathrm{H}_{31} \mathrm{~N}_{3} \mathrm{O}[\mathrm{M}+\mathrm{H}]^{+}=342.2467 \text {; found } 342.2542 .\end{array}$ \\
\hline$\underbrace{+N^{\prime}}_{13 \mathrm{C}}$ & $\begin{array}{l}\text { N-((6-(azepan-1-yl)pyridin-3-yl)methyl)adamantan-1-amine (13c) } \\
{ }^{1} \mathrm{H} \mathrm{NMR}(400 \mathrm{MHz}, \text { Chloroform-d }) \delta 8.23(\mathrm{~d}, J=2.5 \mathrm{~Hz}, 1 \mathrm{H}), 7.93- \\
7.89(\mathrm{dd}, J=8.9,2.5 \mathrm{~Hz}, 1 \mathrm{H}), 6.49(\mathrm{~d}, J=8.9 \mathrm{~Hz}, 1 \mathrm{H}), 3.85(\mathrm{~s}, 2 \mathrm{H}), 3.54 \\
(\mathrm{t}, J=5.9 \mathrm{~Hz}, 4 \mathrm{H}), 3.19-2.94(\mathrm{~m}, 1 \mathrm{H}), 2.17(\mathrm{~s}, 3 \mathrm{H}), 2.12(\mathrm{~s}, 4 \mathrm{H}), 1.78- \\
1.66(\mathrm{~m}, 12 \mathrm{H}), 1.58-1.49(\mathrm{~m}, 4 \mathrm{H}) .{ }^{13} \mathrm{C} \mathrm{NMR}(125 \mathrm{MHz}, \mathrm{Chloroform}-\mathrm{d}) \\
\delta 151.74,145.95,139.72,111.09,58.45,49.77,39.69,38.40,35.68, \\
29.23,26.75,26.62 . \mathrm{C}_{22} \mathrm{H}_{33} \mathrm{~N}_{3} \mathrm{EI}-\mathrm{MS}: \mathrm{m} / \mathrm{z}\left(\mathrm{M}+\mathrm{H}^{+}\right): 340.3(\text { calculated }), \\
340.0 \text { (found). Yield } 80 \% . \mathrm{HRMS}(\mathrm{ESI}) \mathrm{m} / \mathrm{z} \text { calculated for } \mathrm{C}_{22} \mathrm{H}_{33} \mathrm{~N}_{3} \\
{[\mathrm{M}+\mathrm{H}]^{+}=340.2674 ; \text { found } 340.2746 .}\end{array}$ \\
\hline
\end{tabular}




\begin{tabular}{|c|c|}
\hline & $\begin{array}{l}\text { N-((6-(azocan-1-yl)pyridin-3-yl)methyl)adamantan-1-amine (13d) } \\
{ }^{1} \mathrm{H} \mathrm{NMR}(400 \mathrm{MHz} \text {, Chloroform-d) } \delta 7.94(\mathrm{dd}, J=3.1,0.7 \mathrm{~Hz}, 1 \mathrm{H}), 7.23 \\
(\mathrm{d}, J=8.7 \mathrm{~Hz}, 1 \mathrm{H}), 6.96(\mathrm{dd}, J=8.7,3.0 \mathrm{~Hz}, 1 \mathrm{H}), 4.21(\mathrm{~s}, 2 \mathrm{H}), 3.60- \\
3.36(\mathrm{~m}, 4 \mathrm{H}), 2.22(\mathrm{~s}, 3 \mathrm{H}), 2.08(\mathrm{~d}, J=3.0 \mathrm{~Hz}, 6 \mathrm{H}), 1.82-1.47(\mathrm{~m}, 16 \mathrm{H}) \text {. } \\
{ }^{13} \mathrm{C} \mathrm{NMR}(125 \mathrm{MHz}, \mathrm{Chloroform}-\mathrm{d}) \delta 146.33,131.35,129.56,125.28, \\
124.20,60.09,51.16,39.23,38.40,35.60,29.32,26.99,26.65,25.74 . \\
\mathrm{C}_{23} \mathrm{H}_{35} \mathrm{~N}_{3} \mathrm{EI}-\mathrm{MS}: \mathrm{m} / \mathrm{z}\left(\mathrm{M}+\mathrm{H}^{+}\right): 354.3(\text { calculated }), 354.0 \text { (found). Yield } \\
75 \% \text {. HRMS }(\mathrm{ESI}) \mathrm{m} / \mathrm{z} \text { calculated for } \mathrm{C}_{23} \mathrm{H}_{35} \mathrm{~N}_{3}[\mathrm{M}+\mathrm{H}]^{+}=354.2831 ; \\
\text { found 354.2904. }\end{array}$ \\
\hline & $\begin{array}{l}\text { 3-((6-(azocan-1-yl)pyridin-3-yl)methyl)amino)adamantan-1-ol (13e) } \\
{ }^{1} \mathrm{H} \mathrm{NMR}(400 \mathrm{MHz} \text {, Chloroform-d) } \delta 8.32(\mathrm{~d}, J=2.4 \mathrm{~Hz}, 1 \mathrm{H}), 7.60(\mathrm{dd}, J \\
=8.1,2.5 \mathrm{~Hz}, 1 \mathrm{H}), 7.43(\mathrm{~d}, J=8.1 \mathrm{~Hz}, 1 \mathrm{H}), 3.75(\mathrm{~s}, 2 \mathrm{H}), 2.83(\mathrm{t}, J=4.2 \\
\mathrm{Hz}, 4 \mathrm{H}), 2.37-2.22(\mathrm{~m}, 2 \mathrm{H}), 1.63(\mathrm{~d}, J=24.1 \mathrm{~Hz}, 22 \mathrm{H}) . \mathrm{C}_{23} \mathrm{H}_{35} \mathrm{~N}_{3} \mathrm{O} \text { EI- } \\
\mathrm{MS}: \mathrm{m} / \mathrm{z}\left(\mathrm{M}+\mathrm{H}^{+}\right): 370.3 \text { (calculated), } 370.0 \text { (found). Yield } 80 \% \text {. HRMS } \\
(\mathrm{ESI}) \mathrm{m} / \mathrm{z} \text { calculated for } \mathrm{C}_{23} \mathrm{H}_{35} \mathrm{~N}_{3} \mathrm{O}[\mathrm{M}+\mathrm{H}]^{+}=370.2780 \text {; found } \\
370.2855 \text {. }\end{array}$ \\
\hline 4 & $\begin{array}{l}\text { N-((5-bromopyridin-2-yl)methyl)adamantan-1-amine (15) } \\
{ }^{1} \mathrm{H} \text { NMR }(400 \mathrm{MHz}, \text { Chloroform-d) } \delta 8.58(\mathrm{dd}, J=2.4,0.7 \mathrm{~Hz}, 1 \mathrm{H}), 7.74 \\
(\mathrm{dd}, J=8.3,2.4 \mathrm{~Hz}, 1 \mathrm{H}), 7.38-7.24(\mathrm{~m}, 1 \mathrm{H}), 3.90(\mathrm{~s}, 2 \mathrm{H}), 2.35(\mathrm{br}, 1 \mathrm{H}), \\
2.10-2.07(\mathrm{~s}, 3 \mathrm{H}), 1.75-1.59(\mathrm{~m}, 12 \mathrm{H}) .{ }^{13} \mathrm{C} \mathrm{NMR}(125 \mathrm{MHz}, \\
\text { Chloroform-d) } \delta 150.22,149.98,140.41,124.39,120.98,58.25,42.76, \\
\text { 39.07, 35.61, 29.15. C16H21BrN2 EI-MS: } \mathrm{m} / \mathrm{z}\left(\mathrm{M}+\mathrm{H}^{+}\right): 321.1 \\
\text { (calculated), 321.0 (found). Yield } 84 \% \text {. HRMS }(\mathrm{ESI}) \mathrm{m} / \mathrm{z} \text { calculated for } \\
\text { C16H21BrN2 [M+H] }{ }^{+}=321.0888 ; \text { found } 321.0960 .\end{array}$ \\
\hline$\underbrace{}_{16 \mathrm{a}}$ & $\begin{array}{l}\text { N-((5-(piperidin-1-yl)pyridin-2-yl)methyl)adamantan-1-amine (16a) } \\
{ }^{1} \mathrm{H} \mathrm{NMR}(400 \mathrm{MHz} \text {, Chloroform-d) } \delta 8.15(\mathrm{~d}, J=2.8 \mathrm{~Hz}, 1 \mathrm{H}), 7.34(\mathrm{~d}, J \\
=8.7 \mathrm{~Hz}, 1 \mathrm{H}), 7.24-7.19(\mathrm{~m}, 1 \mathrm{H}), 4.21(\mathrm{~s}, 2 \mathrm{H}), 3.23-3.16(\mathrm{~m}, J=4.8 \\
\mathrm{Hz}, 4 \mathrm{H}), 3.09-3.04(\mathrm{~m}, 1 \mathrm{H}), 2.18(\mathrm{~s}, 3 \mathrm{H}), 2.08-1.98(\mathrm{~m}, 6 \mathrm{H}), 1.77-1.61 \\
(\mathrm{~m}, 12 \mathrm{H}) .{ }^{13} \mathrm{C} \mathrm{NMR}(125 \mathrm{MHz}, \mathrm{Chloroform}-\mathrm{d}) \delta 147.73,136.63,123.94, \\
122.85,58.08,49.52,42.43,39.22,35.66,29.16,25.49,24.10 . \mathrm{C}_{21} \mathrm{H}_{31} \mathrm{~N}_{3} \\
\text { EI-MS: } \mathrm{m} / \mathrm{z}\left(\mathrm{M}+\mathrm{H}^{+}\right): 326.2(\text { calculated }), 326.0(\text { found }) . \text { Yield } 81 \% \text {. } \\
\text { HRMS }(\mathrm{ESI}) \mathrm{m} / \mathrm{z} \text { calculated for } \mathrm{C}_{21} \mathrm{H}_{31} \mathrm{~N}_{3}[\mathrm{M}+\mathrm{H}]^{+}=326.2518 ; \text { found } \\
\text { 326.2590. }\end{array}$ \\
\hline
\end{tabular}




\begin{tabular}{|c|c|}
\hline (16b & $\begin{array}{l}\text { 3-(((5-(piperidin-1-yl)pyridin-2-yl)methyl)amino)adamantan-1-ol } \\
(16 b){ }^{1} \mathrm{H} \text { NMR }(400 \mathrm{MHz}, \mathrm{Chloroform}-\mathrm{d}) \delta 8.15(\mathrm{dd}, J=2.9,0.7 \mathrm{~Hz}, \\
1 \mathrm{H}), 7.42(\mathrm{dd}, J=8.6,0.6 \mathrm{~Hz}, 1 \mathrm{H}), 7.21(\mathrm{dd}, J=8.7,2.9 \mathrm{~Hz}, 1 \mathrm{H}), 4.27(\mathrm{~s}, \\
2 \mathrm{H}), 3.25-3.18(\mathrm{~m}, 4 \mathrm{H}), 3.15-3.11(\mathrm{~m}, 1 \mathrm{H}), 2.37(\mathrm{~s}, 2 \mathrm{H}), 2.20-2.14(\mathrm{~m}, \\
1 \mathrm{H}), 2.03-1.88(\mathrm{~m}, 5 \mathrm{H}), 1.83-1.54(\mathrm{~m}, 14 \mathrm{H}) .{ }^{13} \mathrm{C} \mathrm{NMR}(125 \mathrm{MHz}, \\
\text { Chloroform-d) } \delta 148.26,131.46,130.85,127.89,126.99,69.43,61.58, \\
\text { 48.12, } 45.61,44.79,43.21,39.76,37.07,34.12,30.33,24.88,23.61 \text {, } \\
\text { 22.45. } \mathrm{C}_{21} \mathrm{H}_{31} \mathrm{~N}_{3} \mathrm{O} \text { EI-MS: } \mathrm{m} / \mathrm{z}\left(\mathrm{M}+\mathrm{H}^{+}\right): 342.2(\text { calculated }), 342.0(\text { found }) \text {. } \\
\text { Yield } 79 \% \text {. HRMS }(\mathrm{ESI}) \mathrm{m} / \mathrm{z} \text { calculated for } \mathrm{C}_{21} \mathrm{H}_{31} \mathrm{~N}_{3} \mathrm{O}[\mathrm{M}+\mathrm{H}]^{+}= \\
\text {342.2467; found } 342.2539 .\end{array}$ \\
\hline$\sqrt{16 c}$ & $\begin{array}{l}\text { N-((5-(azepan-1-yl)pyridin-2-yl)methyl)adamantan-1-amine (16c) } \\
{ }^{1} \mathrm{H} \mathrm{NMR}(400 \mathrm{MHz}, \text { Chloroform-d) } \delta 8.08(\mathrm{~d}, J=9.2 \mathrm{~Hz}, 1 \mathrm{H}), 7.81(\mathrm{~d}, J \\
=3.1 \mathrm{~Hz}, 1 \mathrm{H}), 7.44(\mathrm{dd}, J=9.1,3.1 \mathrm{~Hz}, 1 \mathrm{H}), 4.35(\mathrm{~s}, 2 \mathrm{H}), 3.63-3.41(\mathrm{~m}, \\
4 \mathrm{H}), 2.27(\mathrm{~s}, 2 \mathrm{H}), 2.21-2.17(\mathrm{~m}, 6 \mathrm{H}), 1.84(\mathrm{~d}, J=5.9 \mathrm{~Hz}, 4 \mathrm{H}), 1.76(\mathrm{~d}, J \\
=3.0 \mathrm{~Hz}, 6 \mathrm{H}), 1.65-1.61(\mathrm{~m}, 4 \mathrm{H}) .{ }^{13} \mathrm{C} \mathrm{NMR}(100 \mathrm{MHz}, \mathrm{Chloroform}-\mathrm{d}) \\
\delta 146.97,131.20,129.92,124.87,124.29,59.85,49.42,39.23,38.31, \\
35.55,29.26,26.82,26.70 . \mathrm{C}_{22} \mathrm{H}_{33} \mathrm{~N}_{3} \mathrm{EI}-\mathrm{MS}: \mathrm{m} / \mathrm{z}\left(\mathrm{M}+\mathrm{H}^{+}\right): 340.3 \\
(\text { calculated }), 340.0 \text { (found). Yield } 80 \% . \mathrm{HRMS}(\mathrm{ESI}) \mathrm{m} / \mathrm{z} \text { calculated for } \\
\mathrm{C}_{22} \mathrm{H}_{33} \mathrm{~N}_{3}[\mathrm{M}+\mathrm{H}]^{+}=340.2674 \text {; found } 340.2749 .\end{array}$ \\
\hline$\underbrace{}_{16 \mathrm{~d}}$ & $\begin{array}{l}\text { 3-(((5-(azepan-1-yl)pyridin-2-yl)methyl)amino)adamantan-1-ol (16d) } \\
{ }^{1} \mathrm{H} \mathrm{NMR}(400 \mathrm{MHz} \text {, Chloroform-d) } \delta 7.97(\mathrm{~d}, J=3.0 \mathrm{~Hz}, 1 \mathrm{H}), 7.26(\mathrm{dd}, J \\
=8.7,0.7 \mathrm{~Hz}, 1 \mathrm{H}), 7.00(\mathrm{dd}, J=8.7,3.0 \mathrm{~Hz}, 1 \mathrm{H}), 4.25(\mathrm{~s}, 2 \mathrm{H}), 3.64- \\
3.38(\mathrm{~m}, 4 \mathrm{H}), 3.38-3.19(\mathrm{~m}, 1 \mathrm{H}), 2.41(\mathrm{~s}, 2 \mathrm{H}), 2.13-2.00(\mathrm{~m}, 6 \mathrm{H}), 1.86 \\
-1.55(\mathrm{~m}, 14 \mathrm{H}) .{ }^{13} \mathrm{C} \mathrm{NMR}(100 \mathrm{MHz}, \mathrm{Chloroform}-\mathrm{d}) \delta 145.08,135.54, \\
131.96,124.50,119.07,69.17,60.59,49.08,46.45,45.98,43.50,43.28, \\
37.67,34.29,30.33,27.30,27.05,27.01,24.94 . \mathrm{C}_{22} \mathrm{H}_{33} \mathrm{~N}_{3} \mathrm{O} \mathrm{EI}-\mathrm{MS}: \mathrm{m} / \mathrm{z} \\
\left(\mathrm{M}+\mathrm{H}^{+}\right): 356.3(\text { calculated }), 356.0 \text { (found). Yield } 75 \% \text {. HRMS }(\mathrm{ESI}) \mathrm{m} / \mathrm{z} \\
\text { calculated for } \mathrm{C}_{22} \mathrm{H}_{33} \mathrm{~N}_{3} \mathrm{O}[\mathrm{M}+\mathrm{H}]^{+}=356.2624 \text {; found } 356.2695 .\end{array}$ \\
\hline$\underbrace{}_{16 e}$ & $\begin{array}{l}\text { N-((5-(azocan-1-yl)pyridin-2-yl)methyl)adamantan-1-amine (16e) } \\
{ }^{1} \mathrm{H} \mathrm{NMR}(400 \mathrm{MHz} \text {, Chloroform-d) } \delta 7.90(\mathrm{dd}, J=3.0,0.6 \mathrm{~Hz}, 1 \mathrm{H}), 7.16 \\
(\mathrm{d}, 3.0 \mathrm{H}, 1 \mathrm{H}), 6.93(\mathrm{dd}, J=8.7,3.0 \mathrm{~Hz}, 1 \mathrm{H}), 4.17(\mathrm{~s}, 2 \mathrm{H}), 3.44-3.39 \\
(\mathrm{~m}, 4 \mathrm{H}), 2.18(\mathrm{~s}, 3 \mathrm{H}), 2.03(\mathrm{~d}, J=2.9 \mathrm{~Hz}, 6 \mathrm{H}), 1.76-1.46(\mathrm{~m}, 16 \mathrm{H}) .{ }^{13} \mathrm{C} \\
\mathrm{NMR}(100 \mathrm{MHz}, \mathrm{Chloroform}-\mathrm{d}) \delta 146.31,131.49,129.72,125.15, \\
124.14,60.08,51.16,39.23,38.41,35.63,29.33,27.01,26.66,25.78 . \\
\mathrm{C}_{23} \mathrm{H}_{35} \mathrm{~N}_{3} \mathrm{EI}-\mathrm{MS}: \mathrm{m} / \mathrm{z}\left(\mathrm{M}+\mathrm{H}^{+}\right): 354.3(\text { calculated }), 354.0 \text { (found). Yield } \\
76 \% \text {. HRMS }(\mathrm{ESI}) \mathrm{m} / \mathrm{z} \text { calculated for } \mathrm{C}_{22} \mathrm{H}_{33} \mathrm{~N}_{3} \mathrm{O}[\mathrm{M}+\mathrm{H}]^{+}=354.2831 ; \\
\text { found } 354.2905 .\end{array}$ \\
\hline
\end{tabular}




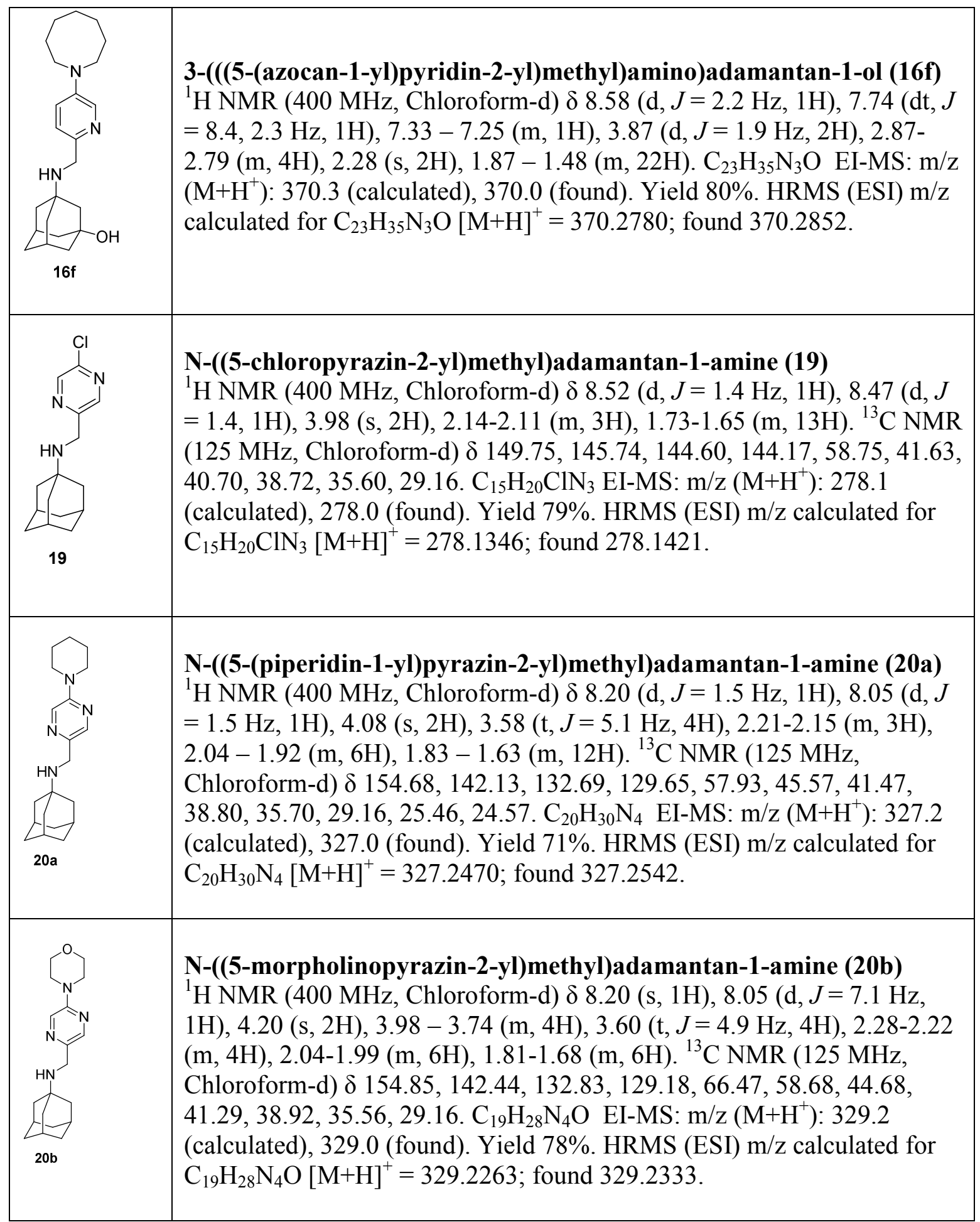




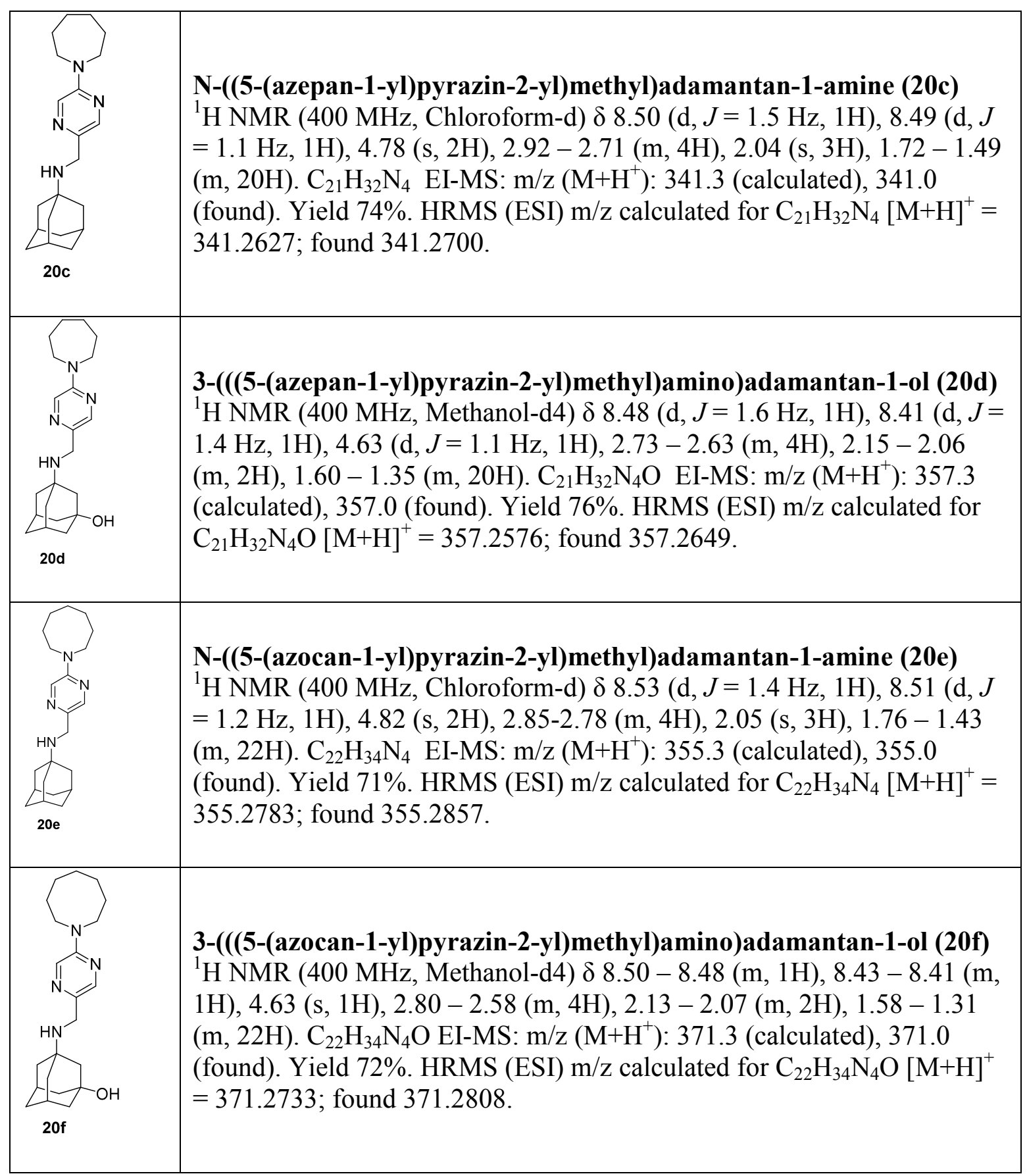

